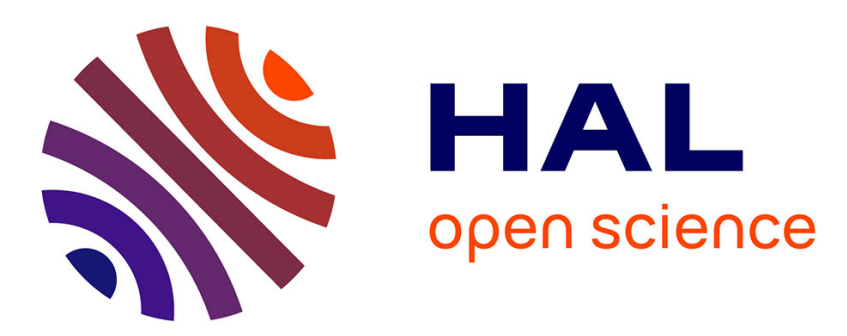

\title{
DÉTECTEUR DE LA PRÉSENCE D'UN LIQUIDE À ONDES ÉLASTIQUES
}

\author{
E. Dieulesaint, O. Legras, D. Royer
}

\section{To cite this version:}

E. Dieulesaint, O. Legras, D. Royer. DÉTECTEUR DE LA PRÉSENCE D'UN LIQUIDE À ONDES ÉLASTIQUES. Journal de Physique Colloques, 1990, 51 (C2), pp.C2-53-C2-56. 10.1051/jphyscol:1990213 . jpa-00230424

\section{HAL Id: jpa-00230424 https://hal.science/jpa-00230424}

Submitted on 1 Jan 1990

HAL is a multi-disciplinary open access archive for the deposit and dissemination of scientific research documents, whether they are published or not. The documents may come from teaching and research institutions in France or abroad, or from public or private research centers.
L'archive ouverte pluridisciplinaire HAL, est destinée au dépôt et à la diffusion de documents scientifiques de niveau recherche, publiés ou non, émanant des établissements d'enseignement et de recherche français ou étrangers, des laboratoires publics ou privés. 
COLLOQUE DE PHYSIQUE

Colloque C2, supplément au $n^{\circ} 2$, Tome 51, Février 1990

ler Congrès Français d'Acoustique 1990

\title{
DÉTECTEUR DE LA PRESENCE D'UN LIQUIDE À ONDES ÉLASTIQUES
}

\author{
E. DIEULESAINT, O. LEGRAS* et D. ROYER \\ Laboratoire d'Acoustoélectricité de I'Université Pierre et Marie Curie, \\ 10 Rue Vauquelin, F-75231 Paris Cedex 05. France \\ * Société Auxitrol, 78 Avenue de la Prospective, F-18025 Bourges Cedex, \\ France
}

\begin{abstract}
Résumé - Deux types de détecteurs basés sur 1'absorption d'ondes élastiques guidées sont décrits. Dans le premier type, détecteur "non intrusif" à ligne à retard, le guide est une partie de 1 a paroi de 1 a cuve. Ce guide, muni de deux transducteurs, est monté en cascade avec un amplificateur de façon à constituer une boucle qui oscille en l'absence de liquide et cesse d'osciller dès que le liquide atteint la zone intertransducteurs. Dans le deuxième type, détecteur intrusif à ligne à échos, le guide est constitué de deux éléments, par exemple cylindriques. L'un est peu sensible à l'environnement, il sert de support mécanique; l'autre est très sensible au contact du liquide. Des trains d'ondes sont périodiquement lancés dans le guide. Ils ne reviennent sous forme d'échos qu'en l'absence de liquide à l'extrémité sensible.

Abstract - Two types of detectors based on absorption of guided elastic waves are described. In the first type, the guide is a part of the wall of the tank. This guide provided with two transducers is connected to an amplifier in order to constitute a loop which oscillates in absence of liquid and stops oscillating when the liquid reaches the zone between transducers. In the second type, the guide is made of two parts. One part is not sensitive to environment and is used as support, the other is very sensitive to the contact of liquid. Wave trains are periodically launched to the guide. They come back as echos in the absence of liquid at the sensitive end.
\end{abstract}

\section{1 - INTRODUCTION}

La connaissance du volume disponible des liquides nécessaires à la vie quotidienne (boissons, hydrocarbures,...) exige la mesure de leurs niveaux dans les réservoirs. Cette mesure est effectuée soit continuement à T'aide d'un capteur, alors appelé jaugeur, soit discrètement, par paliers, à l'aide de capteurs appelés contacteurs ou détecteurs de présence. Les principaux contacteurs actuellement utilisés [1 - 6] sont : - Te flotteur à levier et interrupteur - le condensateur dont une des électrodes est la paroi du réservoir - des dispositifs à ondes élastiques [7] stationnaires (résonateur mécanique : le liquide amortit la vibration) ou progressives: l'émetteur et le récepteur sont disposés dans le réservoir de façon qu'il y ait seulement transmission en présence de liquide.

L'objet de cet article est de décrire deux nouveaux principes de contacteurs à ondes élastiques. Le premier donne lieu à un détecteur "non intrusif" à Tigne à retard[8]. Sa structure à boucle et le mode de propagation exploité le différencient des autres dispositifs évoqués. Le deuxième donne lieu à un détecteur "intrusif" à ligne à échos. Sa structure de guide composite est nouve1 le [9].

\section{2 - DETECTEUR A LIGNE A RETARD}

La figure 1 en illustre le principe. Le transducteur émetteur $E$ engendre un train d'ondes élastiques dans la paroi du réservoir. En l'absence de liquide, ces ondes se propagent sans atténuation vers le transducteur récepteur $R$. Celui-ci les convertit en un signal électrique qui est appliqué à 1 'entrée de 1 'amplificateur. Le gain de l'amplificateur est réglé pour que la tension électrique qu'il fournit à l'émetteur entretienne sa vibration et donc la propagation des ondes. En présence de liquide, ces ondes guidées se convertissent partiellement, au cours de leur propagation, en ondes de volume longitudinales : leur amplitude diminue. La boucle émetteur - ligne à retard (paroi) - récepteur - amplificateur cesse d'osciller dès que le gain de l'amplificateur ne compense plus les pertes. L'arrivée du liquide entre les transducteurs est ainsi révélée par la disparition subite des oscillations, son retrait par leur réapparition. Bans une plaque, les ondes élastiques se propagent suivant un mode symétrique ( $S_{0^{\prime}}$ ) ou suivant un mode antisymétrique $\left(A_{0}\right)$. La figure 2 montre, pour une plaque d'acier, la variation des vitesses de phase et de groupe de ces modes (rapportées à la vitesse $V_{T}$ des ondes transversales 
Fig. I. Détecteur à ligne à retard. En présence de liquide, les ondes guidées se convertissent partiellement en ondes longitudinales. Leur amplitude diminue. Dès que le gain de I'amplificateur ne compense plus cette diminution, la boucle cesse d'osciller.

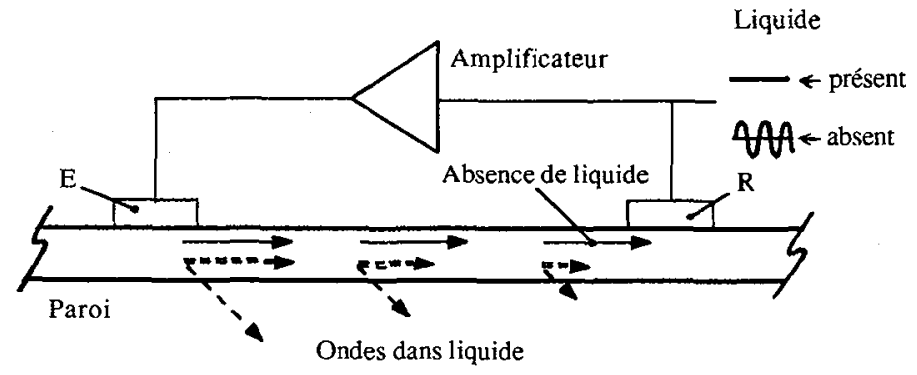
de volume) en fonction du rapport fréquence (f) $x$ épaisseur $(2 h) /$ vitesse $V_{T}$.

Fig. 2. Vitesse de phase (-) et vitesse de groupe (---) des modes de Lamb symétrique $S_{0}$ et antisymétrique Ao dans le cas d'une plaque d'acier d'épaisseur 2h. $V_{m}$ est la vitesse des ondes transversales.

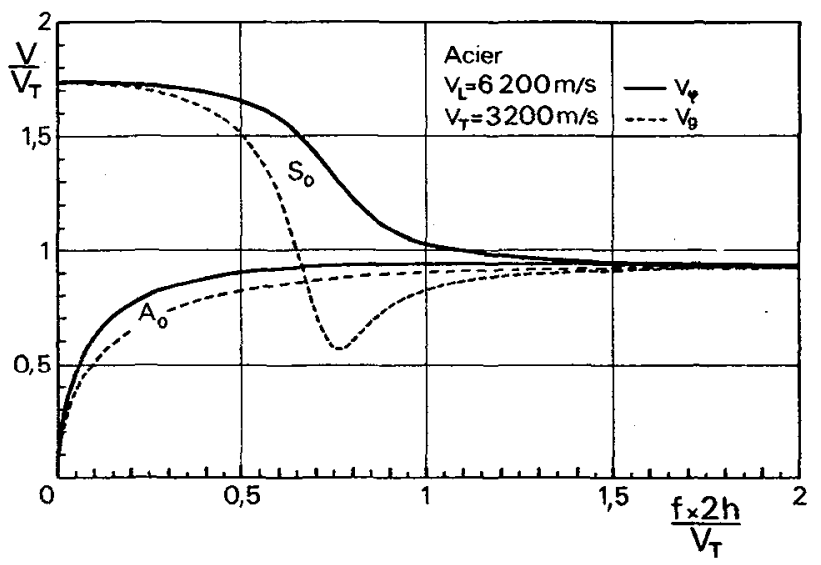

Le point de fonctionnement retenu est le minimum de la vitesse de groupe du mode $S_{0}$, la composante normale du déplacement y est maximale. Ainsi, pour des plaques d'acier d'une épaisseur comprise entre 2,5 et $25 \mathrm{~mm}$, la fréquence de fonctionnement se situe entre 1 et $0,1 \mathrm{MHz}$.

Ce principe a été mis en oeuvre au 1aboratoire, avec des plaques de verre et d'acier, en lançant et détectant. les ondes à l'aide de résonateurs (en céramique piézoélectrique ) fixés sur des prismes (en plexiglass) collés à 1 a plaque. Les résonateurs engendrent des ondes de volume Tongitudinales qui se propagent à la vitesse $V$ et se transforment à $T$ 'interface prisme-plaque en modes de longueur d'onde $\lambda$ tels que

$$
\lambda \cos \alpha=\lambda_{V} \text { avec } \lambda=V_{\phi} / f \text { et } \lambda_{V}=V / f
$$

Fig. 3. Génération des ondes dans la plaque (paroi). Le résonateur excite des ondes longituainales qui se transforment en modes de plaque à I'interface prismeplaque.

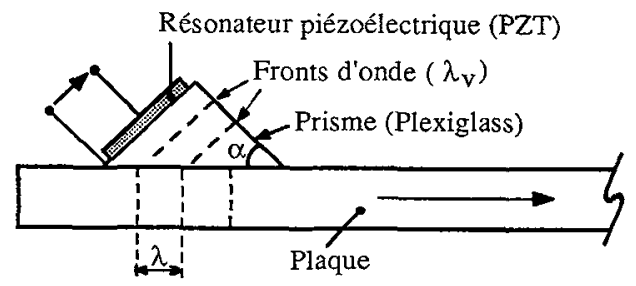

La figure 4 se rapporte à une plaque d'acier d'épaisseur $4 \mathrm{~mm}$, la distance entre prismes est de $15 \mathrm{~cm}$. Les deux premières photographies montrent le signal d'osciliation de la boucle ligne a retard-amplificateur lorsque le gain est - juste égal au gain critique d'accrochage (a) nettement supérieur au gain critique (b). La fréquence d'oscitiation est $580 \mathrm{kHz}$. Dans les. deux cas, la présence d'eau sur la paroi interne de la ligne arrête l'oscillation (c).

\section{3 - DETECTEUR A LIGNE A ECHOS}

Le principe consiste à lancer un train d'ondes élastiques dans un solide et à le détecter après réflexion sur une surface qui entre en contact avec le liquide. Le train d'ondes revient sous forme d'un écho de grande amplitude en 1'absence de liquide et de petite amplitude en présence de liquide. Pour n'avoir pas à adapter les impédances du solide et du liquide, il faut multiplier le nombre de réflexions, c'est à dire guider l'onde. Cependant, quelle que soit la forme 


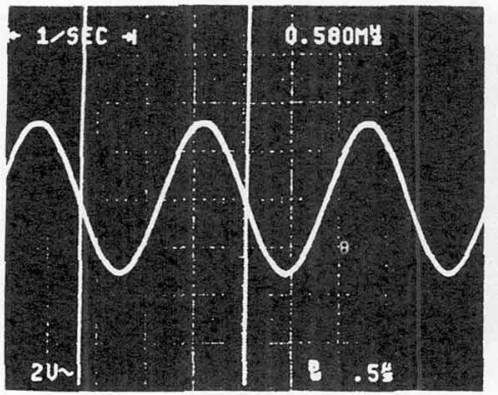

a)

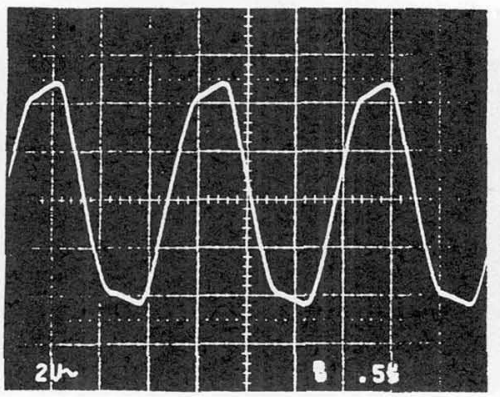

b)

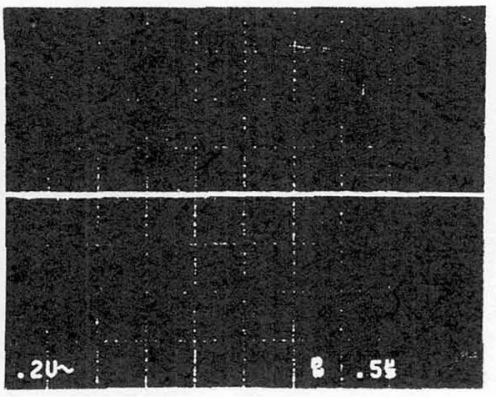

c)

Fig. 4. Oscillation de la boucle ligne à retard - amplificateur avec un gain - égal au gain critique d'accrochage (a) - supérieur au gain critique (b). Quel que soit le gain, la présence d'eau entre les transducteurs arrête l'oscillation (c).

du guide (plaque, cylindre), 11 faut résoudre le problème du support. L'idée est ici d'employer un guide composé de deux tronçons, l'un étant peu sensible aux conditions extérieures et supportant la fixation du dispositif, l'autre étant très sensible au contact du liquide.

Cette idée a été exploitée avec un guide cylindrique et un mode de propagation à composante longitudinale prépondérante. Les courbes de la figure 5 décrivent la variation de la vitesse de groupe de ce mode pour deux cylindres pleins et un cylindre creux en acier. Elles montrent que si 1 'on associe le guide a plein $(\phi: 14 \mathrm{~mm})$ au guide creux $c(\phi i: 12, \phi e: 20 \mathrm{~mm})$, $i 1$ est possible, compte tenu de l'observation faite à propos des modes de plaque, de choisir une fréquence, voisine ici de $100 \mathrm{kHz}$, telle que la partie a soit peu sensible au contact du liquide (dispersion peu marquée) et la partie c soit au contraire très sensible.

Fig. 5. Variation de la vitesse de groupe du mode "Iongitudinal" dans - a) un cylindre d'acier plein ( $\phi$ : $14 \mathrm{~mm}$ ) - b) un cylindre d'acier plein $(\varnothing: 20 \mathrm{~mm})$ - c) un tube $\left(\phi_{i}: 12 \mathrm{~mm}\right.$, $\emptyset_{e}: 20 \mathrm{~mm}$ ).

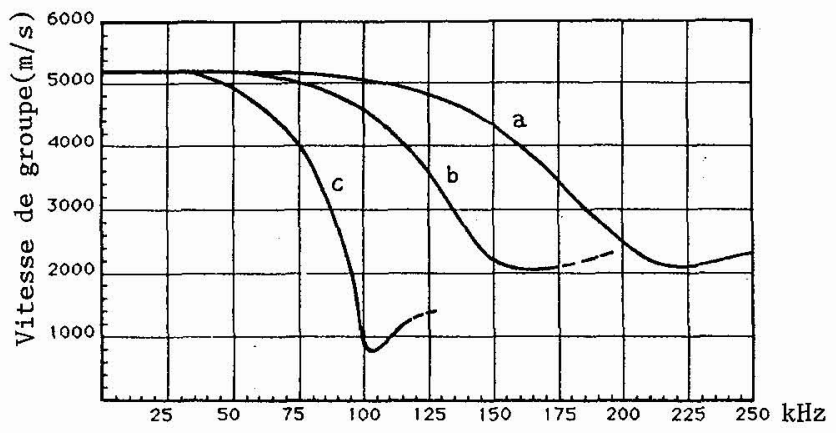

Le guide que nous avons réalisé est représenté sur la figure $6 \mathrm{a}$, la partie A ( $\phi: 14 \mathrm{~mm}$ ) porte le résonateur en céramique qui joue à la fois le rôle d'émetteur et de récepteur et le support (bride d'épaisseur $20 \mathrm{~mm}$ et de diamètre $100 \mathrm{~mm}$ ). La partie $B$ (tube $20 \mathrm{~mm}$ ) est la partie sensible. Une inductance introduit une surtension à l'émission et à la réception et dispense, compte tenu de T'impédance de sortie faíble de T'amplificateur, de portes électroniques. La photographie $6 b$ est le signal détecté, en réponse à une impulsion électrique appliquée au transoducteur, quand la partie $B$ est hors de l'eau. On y remarque l'écho dû à la réflexion à la jonction des deux parties et 1 'écho principal provenant de la partie B. La photographie $6 c$ est le signal détecté quand la partie $B$ est plongée de $5 \mathrm{~cm}$ dans l'eau : I'écho dû à la jonction subsiste intact (i est utilisable comme référence) majs l'écho principal est très atténué. Cette atténuation révèle clairement la présence du liquide.

\section{4 - CONCLUSION.}

Deux nouveaux détecteurs de présence d'un 1iquide à un niveau prédéterminé ont été expérimentés au laboratoire. Le premier, qui a 1'avantage de se disposer à 1'extérieur du réservoir, est un oscillateur à ligne à retard mécanique. Les ondes élastiques se propagent dans cette 1 igne, qui est une partie de la paroi du réservoir, suivant un mode tel que l'oscillation disparaît (réapparait) dès que le liquide, ici de $1^{1}$ eau, pénètre dans (se retire de) la zone intertransducteurs. Le principe s'applique à des parois minces ( $1 \mathrm{~mm}$ ) ou épaisses (20 mm). La résolution est de l'ordre du millimètre. Le fonctionnement, par tout ou rien, dans lequel intervient une condition non seulement sur l'amplitude mais aussi sur la phase, est très sûr. 
Le deuxième détecteur est un guide composite utilisé comme une ligne à échos. L'écho $n^{2}$ est observé qu'en l'absence du liquide. Dans le dispositif décrit, la partie sensible est un tube, cependant, nous avons vérifié qu'il était possible, comme l'indiquent les courbes de dispersion de la figure 5, d'utiliser, au lieu du tube, un cylindre plein. Ce détecteur, muni d'une bride peut, a priori, avantageusement, remplacer un détecteur à flotteur.

\section{5 - REMERCIEMENTS}

Ce travail a été mené, sous contrat ANVAR, en coopération avec la Société AUXITROL. Les auteurs tiennent à remercier M. Plicque de 1 'ANVAR, MM. M. Rogueda et R. Vergnes, directeurs dans cette société et leurs collaborateurs B. Fideler, E.Collet et G. Brault.

\section{6 - REFERENCES}

1 - F. DEMARLES ET A. CORBISIER, "Niveau", Techniques de l'Ingénieur R2010 (1986).

2 - G. ASCH, "Les Capteurs en Instrumentation Industrielle". Dunod, Paris (1982).

3 - Catalogue de la Société AUXITROL (1, rue d'Anjou 92600 Asnières), "Cermat CT 800-70".

4 - Catalogue de la Société VEGA (15, rue du Ried Nordhouse 67150 Erstein), Appareils de mesure de niveau pour l'industrie", 40 (1987).

5 - Catalogue de la Société BESTOBELL MOBREY (190-196 Bath Road, Slough, Berkshire SL1 4DN, U.K) "Mobrey ultrasonic liquid Tevel sensors" (1986).

6 - Catalogue de 1a Société KROHNE (Usine des Ors, B.P. 98, 26103 Romans) "Détecteur de niveau LS $300^{\prime \prime}$.

7 - L. C. LYNWWORTH, "U1trasonic measurements for process Controls" Academic Press, San Diego (1989).

8- E. DIEULESAINT, D. ROYER et 0 . LEGRAS, "Dispositif contacteur à ondes élastiques de Lamb pour la détection de la présence d'un liquide à un niveau prédéterminé" ${ }^{\text {. Brevet } 88.02962}$ (1988).

9 - E. DIEULESAINT, D. ROYER et 0 . LEGRAS, "Détecteur de la présence d'un liquide à guide composite d'ondes élastiques. Brevet 89.09805 (1989).
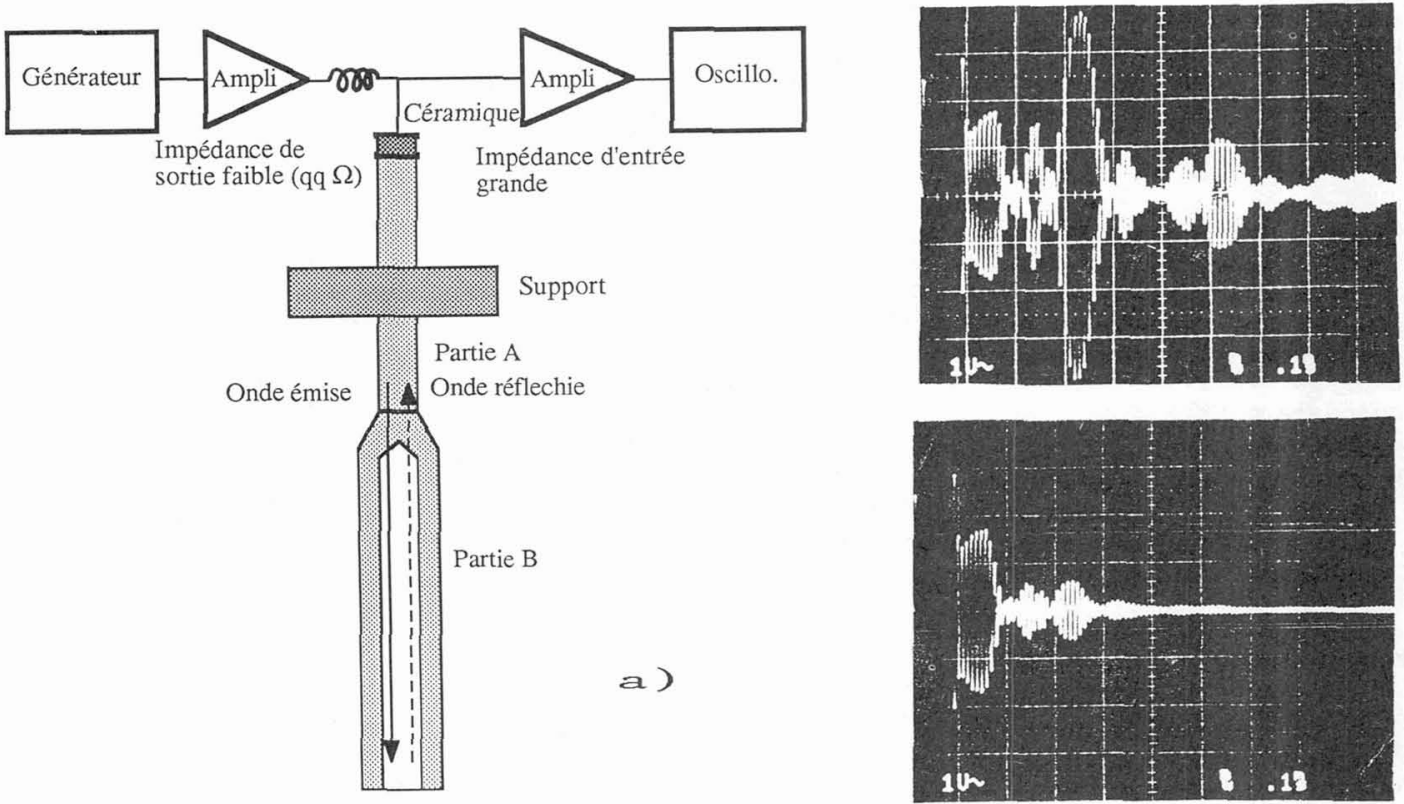

Fig. 6. Détecteur à ligne à échos. a) La ligne est un guide cylindrique comprenant une partie pleine $A$ et une partie creuse $B$, sensible au contact du liquide. Signal détecté quand la partie $B$ est - b) hors de l'eau - c) plongée de $5 \mathrm{~cm}$ dans I'eau. 